\title{
Robust Subspace Blind Channel Estimation for Cyclic Prefixed MIMO OFDM Systems: Algorithm, Identifiability and Performance Analysis
}

\author{
Feifei Gao, Student Member, IEEE, Yonghong Zeng, Senior Member, IEEE, Arumugam Nallanathan, Senior \\ Member, IEEE, and Tung-Sang Ng, Fellow, IEEE
}

\begin{abstract}
A novel subspace (SS) based blind channel estimation method for multi-input, multi-output (MIMO) orthogonal frequency division multiplexing (OFDM) systems is proposed in this work. With an appropriate re-modulation on the received signal blocks, the SS method can be effectively applied to the cyclic prefix (CP) based MIMO-OFDM system when the number of the receive antennas is no less than the number of transmit antennas. These features show great compatibility with the coming fourth generation (4G) wireless communication standards as well as most existing single-input single-output (SISO) OFDM standards, thus allow the proposed algorithm to be conveniently integrated into practical applications. Compared with the traditional SS method, the proposed algorithm exhibits many advantages such as robustness to channel order overestimation, capability of guaranteeing the channel identifiability etc. Analytical expressions for the mean-square error (MSE) and the approximated Cramér-Rao bound (ACRB) of the proposed algorithm are derived in closed forms. Various numerical examples are conducted to corroborate the proposed studies.
\end{abstract}

Index Terms-Blind channel estimation, subspace method, MIMO OFDM, cyclic prefix, identifiability, second order statistics, asymptotical mean square error, Cramér-Rao bound.

\section{INTRODUCTION}

O RTHOGONAL frequency-division multiplexing (OFDM) [1] combined with multiple antennas at both transceiver sides has received considerable attention over the last decade for its promising capability to combat the multipath fading and boost the system capacity, [2], [3]. It also appears as a promising candidate for the coming fourth generation (4G) wireless communications [4].

Several training based channel estimation methods for MIMO OFDM have been developed recently in [5]- [7]. It is shown that the amount of the training increases dramatically with the increment of the number of the transmit antennas [6], [7], which in turn, decreases the system bandwidth efficiency [8]. A substitute is to use blind approaches [9]- [12], which is particularly suitable for packet based transmission, where the channel state information (CSI) is stable for certain number of

Manuscript received April 1, 2007, revised November 1, 2007.

F. Gao is with the Department of Electrical \& Computer Engineering, National University of Singapore (Email: feifeigao@nus.edu.sg).

A. Nallanathan is with the Division of Engineering, King's College London, London, United Kingdom (Email: nallanathan@ieee.org).

Y. Zeng is with Institute for Infocomm Research, A*STAR, Singapore (Email: yhzeng@i2r.a-star.edu.sg).

T.-S. Ng is with the Department of Electrical and Electronic Engineering, The University of Hong Kong, Hong Kong, China (Email: tsng@eee.hku.hk). Digital Object Identifier 10.1109/JSAC.2008.080214. blocks. The blind method only requires the transmission for a short training sequence to remove the estimation ambiguity and thus increases the transmission bandwidth efficiency.

Perhaps the most popular blind algorithm is the so called subspace (SS) based algorithm which was originally developed in [9] for single-input multiple-output (SIMO) frequency selective channels. The SS method has simple structure and achieves good performance, but it meets several difficulties when applied to MIMO OFDM systems [10]- [12]. Firstly, more receive antennas than transmit antennas are required, which seldom holds since the symmetric links play a major role in most wireless transmission standards, e.g. the $2 \times 2$ MIMO for IEEE 802.11n device [13], [14]. Besides, equal number of the transceiver antennas is obviously used in the current SISO OFDM transmission schemes, e.g. IEEE 802.11a standard [15]. Secondly, even for the cases with more receive antennas, the precise knowledge of the channel order must be obtained, which is very difficult in practice. The order over-estimation may produce an ill-conditioned channel matrix which deteriorates the performance of the SS method or sometimes fails the channel estimation.

To solve these problems, a zero-padding (ZP) based MIMO OFDM was suggested in [16]. Instead of using cyclic prefix (CP), consecutive zeros are padded at the end of each OFDM block. This method will be referred to as ZPSOS throughout the paper, where SOS is used for "second order statistics". Although ZPSOS exhibits many advantages, a major problem that prevents its application is the incompatibility to most existing OFDM standards or the future 4G MIMO-OFDM standards [14]. Another way is to use the precoding based algorithm [17], where, by properly designing the coding matrix, the channel information can be directly extracted from the singular-value decomposition (SVD) of the signal covariance matrix. The assumption that the symbols sent from different transmitters are independent and identically distributed (i.i.d.) renders this method both the acceptable performance at low signal-to-noise (SNR) region and the applicability to the multiple-input single-output (MISO) transmission. However, the method meets an error floor at high SNR, and the i.i.d. assumption could not always hold if the transmitted signals are colored, say generated from auto-regressive moving-average (ARMA) processes.

In this paper, we develop a new SS algorithm that is suitable for CP based MIMO OFDM systems by applying 


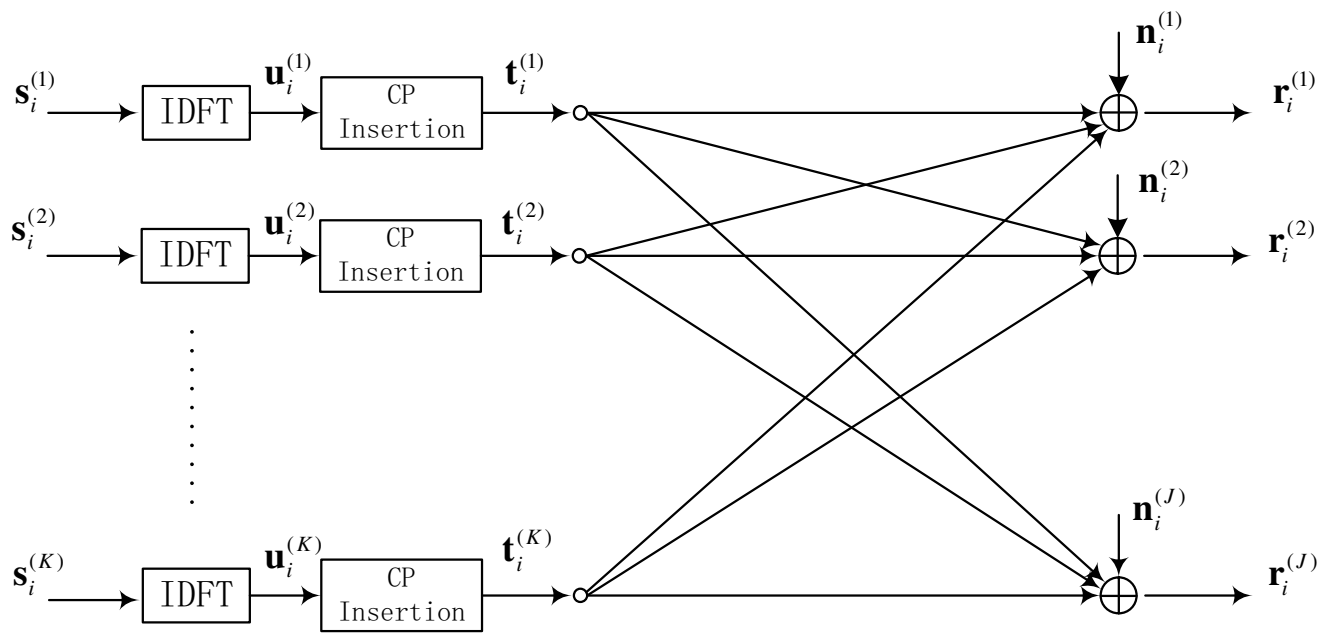

Fig. 1. Multiuser Multiantenna OFDM system.

an appropriate re-modulation on the received signal blocks. The method will be, correspondingly, named as CPSOS. The proposed method possesses all the advantages of ZPSOS but is also compatible with the current (MIMO) OFDM standards. For example, CPSOS is applicable to $K \times J^{1}$ CP-MIMO OFDM systems with $J \geq K$, is robust to channel order overestimation, and could always guarantee the channel identifiability. Consequently, CPSOS is a promising blind channel estimation candidate for MIMO OFDM systems. We also derive both the asymptotical channel estimation mean square error (MSE) and the asymptotically approximated Cramér-Rao bound (ACRB). Interestingly, these two bounds agree with each other.

The paper is organized as follows. Section II presents the system model of MIMO OFDM transmission. Section III describes the proposed algorithm and addresses the related issues. Section IV derives the asymptotical performance analysis of the proposed algorithm. In Section V, we provide the numerical results. Finally, conclusions are drawn in Section VI and related proofs are given in the Appendices.

Notation: vectors and matrices are boldface small and capital letters; the transpose, complex conjugate, Hermitian, inverse, and pseudo-inverse of matrix $\mathbf{A}$ are denoted by $\mathbf{A}^{T}$, $\mathbf{A}^{*}, \mathbf{A}^{H}, \mathbf{A}^{-1}$ and $\mathbf{A}^{\dagger}$, respectively; $\operatorname{tr}(\mathbf{A})$ and $\|\mathbf{A}\|_{F}$ are the trace and the Frobenius norm of $\mathbf{A}$; $\operatorname{diag}\{\mathbf{a}\}$ denotes a diagonal matrix with the diagonal element extracted from a; $\operatorname{vec}(\mathbf{A})$ performs the standard vectorization on the matrix $\mathbf{A} ; \otimes$ stands for the Kronecker product; $\mathbf{I}_{K}$ is the $K \times K$ identity matrix; $\mathrm{E}\{\cdot\}$ denotes the statistical expectation. The MATLAB notations for rows and columns are used. For example, $\mathbf{A}(:, m)$ represents the $m$ th column of the matrix A.

\section{SyStem Model OF MIMO OFDM}

A MIMO system with $K$ transmitters and $J$ receivers is shown in Fig. 1. The information symbols are first divided into $K$ streams and each stream will be grouped into blocks of

\footnotetext{
${ }^{1} \mathrm{~K}$ transmit antennas, $J$ receive antennas
}

length $N$, followed by the normalized inverse discrete Fourier transformation (IDFT). Let

$$
\begin{aligned}
\mathbf{s}_{i}^{(k)}=\left[s_{i}^{(k)}(0), s_{i}^{(k)}(1), \ldots, s_{i}^{(k)}(N-1)\right]^{T} & \\
k & =1,2, \ldots, K, \quad i=0,1, \ldots, M
\end{aligned}
$$

be the $i$ th information block at the $k$ th transmitter and $\mathbf{u}_{i}^{(k)}=$ $\left[u_{i}^{(k)}(0), u_{i}^{(k)}(1), \ldots, u_{i}^{(k)}(N-1)\right]^{T}$ be the normalized IDFT of $\mathbf{s}_{i}^{(k)}$. After the CP insertion, the overall time domain block from the $k$ th transmitter is

$$
\mathbf{t}_{i}^{(k)}=\left[\begin{array}{l}
\mathbf{u}_{i, L}^{(k)} \\
\mathbf{u}_{i}^{(k)}
\end{array}\right]=\mathbf{T}_{\mathrm{cp}} \mathbf{u}_{i}^{(k)}
$$

where $\mathbf{u}_{i, L}^{(k)}$ is the CP that contains the last $L$ entries of $\mathbf{u}_{i}^{(k)}$, and $\mathbf{T}_{\mathrm{cp}}$ is the corresponding $\mathrm{CP}$ insertion matrix. Let

$$
\mathbf{h}^{(j, k)}=\left[h^{(j, k)}(0), h^{(j, k)}(1), \ldots, h^{(j, k)}\left(L_{j, k}\right)\right]^{T}
$$

be the channel impulse response (CIR) from transmitter $k$ to receiver $j$, where $L_{j, k}$ is the corresponding channel order and is uniformly upper bounded by $L$. For convenience, we will pad $L-L_{j, k}$ zeros at the end of $\mathbf{h}^{(j, k)}$ such that they all have a length of $L$. In other words, the channel order over estimation is taken into account in the model here. Assuming perfect synchronization, the received $i$ th block (of length $N+L$ ) on the $j$ th receiver is then represented by

$$
\mathbf{r}_{i}^{(j)}=\sum_{k=1}^{K} \boldsymbol{H}\left(\mathbf{h}^{(j, k)}\right)\left[\begin{array}{c}
\mathbf{u}_{i-1, L}^{(k)} \\
\mathbf{t}_{i}^{(k)}
\end{array}\right]+\mathbf{n}_{i}^{(j)}
$$

where $\mathcal{H}(\cdot)$ is the operation with respect to the argument inside the bracket

$$
\begin{aligned}
& \mathcal{H}\left(\mathbf{h}^{(j, k)}\right)= \\
& \underbrace{\left[\begin{array}{ccccc}
h^{(j, k)}(L) & \ldots & h^{(j, k)}(0) & \ldots & 0 \\
\vdots & \ddots & \ddots & \ddots & \vdots \\
0 & \ldots & h^{(j, k)}(L) & \ldots & h^{(j, k)}(0)
\end{array}\right]}_{(N+2 L) \text { blocks }}\}(N+L) \text { blocks }
\end{aligned}
$$

and

$$
\mathbf{n}_{i}^{(j)}=\left[n_{i}^{(j)}(0), n_{i}^{(j)}(1), \ldots, n_{i}^{(j)}(N+L-1)\right]^{T}
$$


is the $i$ th noise block on the $j$ th receiver whose elements are zero mean complex Gaussian random variables with the variance $\sigma_{n}^{2}$ and are both spatially and temporally independent from each other. For future use, we divide $\mathbf{r}_{i}^{(j)}$ into two parts as $\mathbf{r}_{i}^{(j)}=\left[\left(\mathbf{x}_{i, L}^{(j)}\right)^{T},\left(\mathbf{x}_{i}^{(j)}\right)^{T}\right]^{T}$ where $\mathbf{x}_{i, L}^{(j)}$ and $\mathbf{x}_{i}^{(j)}$ have the structures

$$
\begin{aligned}
& \mathbf{x}_{i, L}^{(j)}=\left[x_{i, L}^{(j)}(0), x_{i, L}^{(j)}(1), \ldots, x_{i, L}^{(j)}(L-1)\right]^{T} \\
& \mathbf{x}_{i}^{(j)}=\left[x_{i}^{(j)}(0), x_{i}^{(j)}(1), \ldots, x_{i}^{(j)}(N-1)\right]^{T}
\end{aligned}
$$

respectively. We then group the transmitted and received signals on the same time slot by defining

$$
\begin{aligned}
& \mathbf{u}_{i}(n)=\left[u_{i}^{(1)}(n), u_{i}^{(2)}(n), \ldots, u_{i}^{(K)}(n)\right]^{T}, \quad n=0, \ldots, N-1 \\
& \mathbf{x}_{i, L}(l)=\left[x_{i, L}^{(1)}(l), x_{i, L}^{(2)}(l), \ldots, x_{i, L}^{(J)}(l)\right]^{T}, l=0, \ldots L-1(8 \mathrm{~b}) \\
& \mathbf{x}_{i}(n)=\left[x_{i}^{(1)}(n), x_{i}^{(2)}(n), \ldots, x_{i}^{(J)}(n)\right]^{T} \\
& \mathbf{h}^{(k)}(q)=\left[h^{(1, k)}(q), h^{(2, k)}(q), \ldots, h^{(J, k)}(q)\right]^{T}, \\
& \mathbf{H}(8 \mathrm{c}) \\
& \mathbf{H}(q)=\left[\mathbf{h}^{(1)}(q), \mathbf{h}^{(2)}(q), \ldots, \mathbf{h}^{(K)}(q)\right] \\
& \mathbf{n}_{i}(p)=\left[n_{i}^{(1)}(p), n_{i}^{(2)}(p), \ldots, n_{i}^{(J)}(p)\right]^{T}, \\
& \quad p=0, \ldots, N+L-1 \\
& \mathbf{x}_{i}=\left[\mathbf{x}_{i}^{T}(0), \mathbf{x}_{i}^{T}(1), \ldots, \mathbf{x}_{i}^{T}(N-1)\right]^{T} \\
& \mathbf{x}_{i, L}=\left[\mathbf{x}_{i, L}^{T}(0), \mathbf{x}_{i, L}^{T}(1), \ldots, \mathbf{x}_{i, L}^{T}(L-1)\right]^{T} \\
& \mathbf{u}_{i}=\left[\mathbf{u}_{i}^{T}(0), \mathbf{u}_{i}^{T}(1), \ldots, \mathbf{u}_{i}^{T}(N-1)\right]^{T} \\
& \mathbf{u}_{i, L}=\left[\mathbf{u}_{i}^{T}(N-L-1), \mathbf{u}_{i}^{T}(N-L), \ldots, \mathbf{u}_{i}^{T}(N-1)\right]^{T}(8 \mathrm{j}) \\
& \mathbf{t}_{i}=\left[\mathbf{u}_{i, L}^{T}, \mathbf{u}_{i}^{T}\right]^{T} \\
& \mathbf{n}_{i}=\left[\mathbf{n}_{i}^{T}(0), \mathbf{n}_{i}^{T}(1), \ldots, \mathbf{n}_{i}^{T}(N+L-1)\right] \\
& \mathbf{H}=\left[\mathbf{H}^{T}(0), \mathbf{H}^{T}(1), \ldots, \mathbf{H}^{T}(L)\right]^{T} .
\end{aligned}
$$

The signal blocks from all the $J$ receivers, after proper entry permutation, can be re-expressed as

$$
\begin{aligned}
\mathbf{r}_{i} & =\left[\begin{array}{c}
\mathbf{x}_{i, L} \\
\mathbf{x}_{i}
\end{array}\right]=\mathcal{H}(\mathbf{H})\left[\begin{array}{c}
\mathbf{u}_{i-1, L} \\
\mathbf{t}_{i}
\end{array}\right]+\mathbf{n}_{i} \\
& =\mathcal{H}(\mathbf{H}) \mathcal{T}_{\mathrm{cp}}\left[\begin{array}{c}
\mathbf{u}_{i-1, L} \\
\mathbf{u}_{i}
\end{array}\right]+\mathbf{n}_{i} .
\end{aligned}
$$

where the structure of $\mathcal{H}(\mathbf{H})$ can be referred to (4) and $\mathcal{T}_{\mathrm{cp}}$ is the corresponding $K(N+2 L) \times K(N+L)$ matrix with the following form

$$
\mathcal{T}_{\mathrm{cp}}=\left[\begin{array}{cr}
\mathbf{I}_{K L} & \mathbf{0}_{K L \times K N} \\
\mathbf{0}_{K L \times K N} & \mathbf{I}_{K L} \\
\mathbf{0}_{K N \times K L} & \mathbf{I}_{K N}
\end{array}\right] .
$$

The SS method could be applied to (9) and the identifiability could be guaranteed if

1) The $J(N+L) \times K(N+L)$ matrix $\mathcal{H}(\mathbf{H}) \mathcal{T}_{\text {cp }}$ is tall.

2) Matrix $\mathcal{H}(\mathbf{H}) \mathcal{T}_{\text {cp }}$ is full rank.

3) Span of $\mathcal{H}(\overline{\mathbf{H}}) \mathcal{T}_{\text {cp }}$ equals to span of $\mathcal{H}(\mathbf{H}) \mathcal{T}_{\text {cp }}$ if and only if $\overline{\mathbf{H}}=\mathbf{H B}$, where $\mathbf{B}$ is an unknown constant matrix.

The first condition is satisfied only if $J>K$. Clearly, the direct modeling on the received signals is not applicable to the scenarios with $J=K$, which includes both the popular SISO
OFDM in IEEE 802.11a [15] and the $2 \times 2$ MIMO OFDM in IEEE 802.11n [14]. To the best of authors' knowledge, the second and the third conditions have not been studied yet in the existing literature. One obvious example that breaks condition 2), 3) is when $\mathbf{H}(L)=\ldots=\mathbf{H}(1)=\mathbf{0}$ but $\mathbf{H}(0)$ is full column rank. In this case, the matrix $\mathcal{H}(\mathbf{H}) \mathcal{T}_{\text {cp }}$ becomes singular.

Remark: Although there do exist the identification study for $\mathcal{H}(\mathbf{H})$ under $J>K[18]$, the appearance of the precoding matrix $\mathcal{T}_{\text {cp }}$ breaks the convolutive property between channel and information symbols. Consequently, the result in [18] cannot be directly applied to the discussion on $\mathcal{H}(\mathbf{H}) \mathcal{T}_{\text {cp }}$.

\section{Proposed Algorithm And The Related Issues}

\section{A. System Re-Modulation}

We find that, by properly remodulating the received signal block, the system model (9) could be converted to the one similar to ZPSOS model proposed in [16]. This enlightens us that the robust property of ZPSOS, e.g. applicability to equal transceiver antenna scenario, robustness to channel order over estimation and guarantee of the channel identifiability, could possibly be inherited after the re-modulation.

Let us first divide the noise vector $\mathbf{n}_{i}$ into two components as $\mathbf{n}_{i 1}=\mathbf{n}_{i}(1: J L)$, and $\mathbf{n}_{i 2}=\mathbf{n}_{i}(J L+1: J(N+L))$. Construct a new vector $\tilde{\mathbf{r}}_{i}=\left[\mathbf{x}_{i-1}^{T}, \mathbf{x}_{i, L}^{T}\right]^{T}$, which could be expressed as

$$
\tilde{\mathbf{r}}_{i}=\mathcal{H}(\mathbf{H})\left[\begin{array}{l}
\mathbf{t}_{i-1} \\
\mathbf{u}_{i, L}
\end{array}\right]+\left[\begin{array}{c}
\mathbf{n}_{(i-1) 2} \\
\mathbf{n}_{i 1}
\end{array}\right]
$$

It can be verified that

$$
\begin{aligned}
\mathbf{z}_{i} & \triangleq \mathbf{r}_{i}-\tilde{\mathbf{r}}_{i} \\
& =\mathcal{H}(\mathbf{H})\left(\left[\begin{array}{c}
\mathbf{u}_{i-1, L} \\
\mathbf{t}_{i}
\end{array}\right]-\left[\begin{array}{c}
\mathbf{t}_{i-1} \\
\mathbf{u}_{i, L}
\end{array}\right]\right)+\underbrace{\left(\mathbf{n}_{i}-\left[\begin{array}{c}
\mathbf{n}_{(i-1) 2} \\
\mathbf{n}_{i 1}
\end{array}\right]\right)}_{\boldsymbol{\eta}_{i}} \\
& =\mathcal{H}(\mathbf{H})\left[\begin{array}{c}
\mathbf{0}_{J L \times 1} \\
\mathbf{d}_{i} \\
\mathbf{0}_{J L \times 1}
\end{array}\right]+\boldsymbol{\eta}_{i} \\
& =\mathcal{G} \mathbf{d}_{i}+\boldsymbol{\eta}_{i}
\end{aligned}
$$

where

$$
\begin{aligned}
& \mathbf{d}_{i}=\mathbf{t}_{i}(1: K N)-\mathbf{u}_{i-1} \\
& =\left[\mathbf{u}_{i, L}^{T}, \mathbf{u}_{i}^{T}(0), \ldots, \mathbf{u}_{i}^{T}(N-L-1)\right]^{T}-\mathbf{u}_{i-1}
\end{aligned}
$$

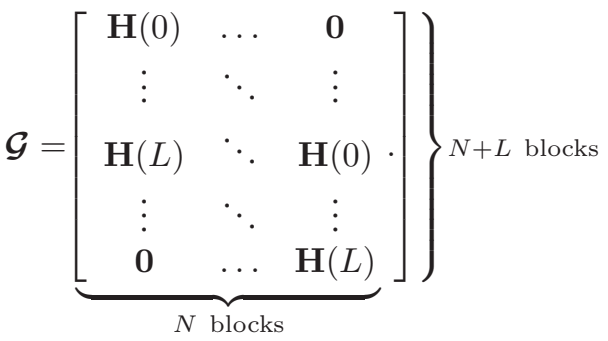

The new noise vector $\boldsymbol{\eta}_{i}$ is colored and has the covariance matrix

$$
\mathbf{R}_{\boldsymbol{\eta}}=\mathrm{E}\left\{\boldsymbol{\eta}_{i} \boldsymbol{\eta}_{i}^{H}\right\}=\sigma_{n}^{2} \mathbf{R}_{w}
$$


with

$$
\mathbf{R}_{w}=\left[\begin{array}{ccc}
2 \mathbf{I}_{J L \times J L} & \mathbf{0} & -\mathbf{I}_{J L \times J L} \\
\mathbf{0} & 2 \mathbf{I}_{J(N-L) \times J(N-L)} & \mathbf{0} \\
-\mathbf{I}_{J L \times J L} & \mathbf{0} & 2 \mathbf{I}_{J L \times J L}
\end{array}\right] .
$$

Although it appears that the noise power in $\boldsymbol{\eta}_{i}$ is increased by a factor of 2, the signal power in $\mathbf{d}_{i}$ is enlarged twice as well. Therefore, the effective SNR for SS algorithm is not changed. Since $\mathcal{G}$ is exactly the same as the channel matrix in ZPSOS [16], we get the following lemma:

Lemma 1 [16]: For $J \geq K$, if there exists an $l \in[0, L]$ such that $\mathbf{H}(l)$ is of full column rank, then $\mathcal{G}$ is of full column rank.

The proof is obvious and is omitted for brevity. The full column rank property of $\mathbf{H}(l)$ is almost surely guaranteed because signal propagation from each of the $K$ transmitters scattered is most likely independent. In the following, we assume that this condition holds. We need to mention that even if $\mathbf{H}(l)$ is not full column rank, it is still possible that $\mathcal{G}$ is of full column rank bearing in mind that Lemma 1 only provides a sufficient condition.

\section{B. Subspace Based Algorithm}

The standard SS method requires the covariance of the noise vector to be a scaled identity matrix. Therefore, we need to whiten the vector $\mathbf{z}_{i}$ by $\mathbf{R}_{w}^{-1 / 2}$ and obtain

$$
\mathbf{y}_{i}=\mathbf{R}_{w}^{-1 / 2} \mathbf{z}_{i}=\underbrace{\mathbf{R}_{w}^{-1 / 2} \mathcal{G}}_{\mathcal{A}} \mathbf{d}_{i}+\tilde{\mathbf{n}}_{i}
$$

where $\tilde{\mathbf{n}}_{i}$ is the $J(N+L) \times 1$ white noise vector whose entries have variance $\sigma_{n}^{2}$. In addition, since $\mathbf{R}_{w}$ is a non-singular matrix, the new channel matrix $\mathcal{A}$ is full column rank if $J \geq$ $K$.

Due to the special structure of $\mathbf{R}_{w}, \mathbf{R}_{w}^{-1 / 2}$ can be calculated as

$$
\mathbf{R}_{w}^{-1 / 2}=\left[\begin{array}{ccc}
c_{1} \mathbf{I}_{J L \times J L} & \mathbf{0} & c_{2} \mathbf{I}_{J L \times J L} \\
\mathbf{0} & \frac{1}{\sqrt{2}} \mathbf{I}_{J(N-L) \times J(N-L)} & \mathbf{0} \\
c_{2} \mathbf{I}_{J L \times J L} & \mathbf{0} & c_{1} \mathbf{I}_{J L \times J L}
\end{array}\right]
$$

where

$$
c_{1}=\sqrt{\frac{2 / 3+\sqrt{1 / 3}}{2}}, \quad c_{2}=\sqrt{\frac{2 / 3-\sqrt{1 / 3}}{2}}
$$

regardless of $J, N, L$. Then the covariance matrix of $\mathbf{y}_{i}$ is derived from

$$
\mathbf{R}=\mathrm{E}\left\{\mathbf{y}_{i} \mathbf{y}_{i}^{H}\right\}=\mathcal{A} \mathbf{R}_{d} \mathcal{A}^{H}+\sigma_{n}^{2} \mathbf{I}_{J(N+L) \times J(N+L)}
$$

where $\mathbf{R}_{d}=\mathrm{E}\left\{\mathbf{d}_{i} \mathbf{d}_{i}^{H}\right\}$ is the source covariance matrix, which should be full rank if no two elements in $\mathbf{d}_{i}$ are fully correlated. This requirement is normally satisfied since the two consecutive blocks $\mathbf{u}_{i}$ and $\mathbf{u}_{i-1}$ are in general not fully correlated. The covariance matrix $\mathbf{R}$ can be eigen-decomposed as

$$
\mathbf{R}=\mathbf{U}_{s} \boldsymbol{\Lambda}_{s} \mathbf{U}_{s}^{H}+\sigma_{n}^{2} \mathbf{U}_{o} \mathbf{U}_{o}^{H}
$$

where $\boldsymbol{\Lambda}_{s}$ is the $K N \times K N$ diagonal matrix and $J(N+$ $L) \times K N$ matrix $\mathbf{U}_{s}$ spans the signal-subspace of $\mathbf{R}$. In turn, $J(N+L) \times(J(N+L)-K N)$ matrix $\mathbf{U}_{o}$ spans the noise-subspace of $\mathbf{R}$. The standard SS method says that the matrix $\mathbf{U}_{o}$ is orthogonal to every column of $\mathcal{A}$. This can be equivalently expressed as

$$
\mathbf{U}_{o}^{H} \mathbf{R}_{w}^{-1 / 2} \mathbf{C}_{n} \mathbf{H}=\mathbf{0}, \quad n=1, \ldots, N
$$

where $\mathbf{C}_{n}$ is the $J(N+L) \times J(L+1)$ Toeplitz matrix with the first column $\mathbf{e}_{(n-1) J+1}$ and $\mathbf{e}_{p}$ is defined as the $p$ th column of $\mathbf{I}_{J(N+L)}$. The first row of $\mathbf{C}_{n}$ is $\left[1, \mathbf{0}_{1 \times(J(L+1)-1)}\right]$ for $n=1$ and is $\mathbf{0}_{1 \times J(L+1)}$ for $n \geq 2$.

Define

$$
\mathcal{K}=\left[\mathbf{C}_{1}^{H} \mathbf{R}_{w}^{-1 / 2} \mathbf{U}_{o}, \mathbf{C}_{2}^{H} \mathbf{R}_{w}^{-1 / 2} \mathbf{U}_{o}, \ldots, \mathbf{C}_{N}^{H} \mathbf{R}_{w}^{-1 / 2} \mathbf{U}_{o}\right] .
$$

The channel matrix $\mathbf{H}$ could be estimated from

$$
\mathcal{K}^{H} \mathbf{H}=\mathbf{0} .
$$

Therefore, the estimate of $\mathbf{H}$, denoted as $\hat{\mathbf{H}}$, is a basis matrix of the orthogonal complement space of $\mathcal{K}$. We will show later that the dimension of the orthogonal complement space of $\mathcal{K}$ is exactly $K$. Therefore, $\hat{\mathbf{H}}$ can be obtained from left singular vectors of $\mathcal{K}$ and is away from the true $\mathbf{H}$ by an unknown matrix $\mathbf{B}$, namely

$$
\hat{\mathbf{H}}=\mathbf{H B} \text {. }
$$

Note that, matrix $\mathbf{B}$ must be full rank since $\hat{\mathbf{H}}$ and $\mathbf{H}$ are all full rank. This matrix ambiguity could easily be resolved by transmitting some training symbols as suggested in [16]. A sketch on the process is provided here. Let matrix $\hat{\mathcal{G}}$ be constructed from $\hat{\mathbf{H}}$ following the same way in (14). We know that

$$
\mathcal{G}=\hat{\mathcal{G}}\left(\mathbf{I}_{N} \otimes \mathbf{B}^{-1}\right)
$$

Therefore,

$$
\mathbf{z}_{i}=\hat{\mathcal{G}}\left(\mathbf{I}_{N} \otimes \mathbf{B}^{-1}\right) \mathbf{d}_{i}+\boldsymbol{\eta}_{i} .
$$

Since $\hat{\mathcal{G}}$ is of full column rank, we can find its pseudo inverse, denoted by $\hat{\mathcal{G}}^{\dagger}$, and define $\breve{\mathbf{z}}_{i}=\hat{\mathcal{G}}^{\dagger} \mathbf{z}_{i}, \breve{\boldsymbol{\eta}}_{i}=\hat{\mathcal{G}}^{\dagger} \boldsymbol{\eta}_{i}$. Then

$$
\breve{\mathbf{z}}_{i}=\left(\mathbf{I}_{N} \otimes \mathbf{B}^{-1}\right) \mathbf{d}_{i}+\breve{\boldsymbol{\eta}}_{i} .
$$

By dividing the vector $\breve{\mathbf{z}}_{i}, \mathbf{d}_{i}$, and $\breve{\boldsymbol{\eta}}_{i}$ into blocks of length $K$, we get

$$
\begin{aligned}
\breve{\mathbf{z}}_{i} & =\left[\breve{\mathbf{z}}_{i}^{T}(0), \breve{\mathbf{z}}_{i}^{T}(1), \ldots, \breve{\mathbf{z}}_{i}^{T}(N-1)\right]^{T} \\
\mathbf{d}_{i} & =\left[\mathbf{d}_{i}^{T}(0), \mathbf{d}_{i}^{T}(1), \ldots, \mathbf{d}_{i}^{T}(N-1)\right]^{T} \\
\breve{\boldsymbol{\eta}}_{i} & =\left[\breve{\boldsymbol{\eta}}_{i}^{T}(0), \breve{\boldsymbol{\eta}}_{i}^{T}(1), \ldots, \breve{\boldsymbol{\eta}}_{i}^{T}(N-1)\right]^{T} .
\end{aligned}
$$

The relationship is now written as

$$
\breve{\mathbf{z}}_{i}(n)=\mathbf{B}^{-1} \mathbf{d}_{i}(n)+\breve{\boldsymbol{\eta}}_{i}(n), \quad n=1, \ldots, N .
$$

Let

$$
\begin{aligned}
\breve{\mathbf{Z}}_{i} & =\left[\breve{\mathbf{z}}_{i}(0), \breve{\mathbf{z}}_{i}(1), \ldots, \breve{\mathbf{z}}_{i}(N-1)\right] \\
\breve{\mathbf{D}} & =\left[\mathbf{d}_{i}(0), \mathbf{d}_{i}(1), \ldots, \mathbf{d}_{i}(N-1)\right] \\
\breve{\Xi}_{i} & =\left[\breve{\boldsymbol{\eta}}_{i}(0), \breve{\boldsymbol{\eta}}_{i}(1), \ldots, \breve{\boldsymbol{\eta}}_{i}(N-1)\right] .
\end{aligned}
$$

Then

$$
\breve{\mathbf{Z}}_{i}=\mathbf{B}^{-1} \breve{\mathbf{D}}+\breve{\Xi}_{i} .
$$

Since the variance of the noise matrix $\breve{\Xi}_{i}$ can be calculated, $\mathbf{B}^{-1}$ can be obtained from standard estimation approach, e.g. the ML detector or the minimum mean square error (MMSE) detector, and the details are omitted here. 


\section{Remarks:}

- To make $\breve{\mathbf{D}}_{i}$ a known matrix, we only need to adjust $\mathbf{u}_{i}$ but keep $\mathbf{u}_{i-1}$ carrying the unknown information.

- Note that the ambiguity could be resolved whenever $\breve{\mathbf{D}}$ is a fat matrix. Therefore, we only need to know $K$ columns of $\breve{\mathbf{D}}_{i}$, which requires $K$ training symbols from each transmit antennas. This amount of training is much smaller than that required by a direct training based channel estimation.

\section{Channel Identifiability and Order Over-Estimation}

Thanks to the proposed re-modulation, the channel matrix $\mathcal{A}$ possesses the similar structure as that in [16], which greatly facilitates the study of the identifiability issue.

Theorem 1: If $\mathbf{H}(0)$ is full column rank, then the matrix $\mathbf{H}$ is uniquely determined by $\operatorname{span}(\mathcal{A})$ subject to a common $K \times K$ non-singular matrix ambiguity on each $\mathbf{H}(l)$.

Proof: For a $K(L+1) \times K$ matrix $\overline{\mathbf{H}}=$ $\left[\overline{\mathbf{H}}^{T}(0), \overline{\mathbf{H}}^{T}(1), \ldots, \overline{\mathbf{H}}^{T}(L)\right]^{T}$, let $\overline{\mathcal{A}}=\mathbf{R}_{w}^{-1 / 2} \overline{\mathcal{G}}$, where $\mathcal{G}$ is constructed from $\overline{\mathbf{H}}$ in a similar way how $\mathcal{G}$ is constructed from $\mathbf{H}$. If $\operatorname{span}(\overline{\mathcal{A}})=\operatorname{span}(\mathcal{A})$, then

$$
\overline{\mathcal{A}}=\mathcal{A P}
$$

where $\mathcal{P}$ is an invertible matrix of dimension $K N \times K N$. Since $\mathbf{R}_{w}^{-1 / 2}$ is a full rank matrix, it is not difficult to derive the following equality:

$$
\overline{\mathcal{G}}=\mathcal{G P} .
$$

Therefore, we know $\operatorname{span}(\overline{\mathcal{G}})=\operatorname{span}(\mathcal{G})$ and there is

$$
\overline{\mathcal{G}}=\mathcal{G B}
$$

where $\mathcal{B}$ is a $K N \times K N$ matrix with the form

$$
\mathcal{B}=\left[\begin{array}{cccc}
\mathbf{B}_{11} & \mathbf{B}_{12} & \ldots & \mathbf{B}_{1 N} \\
\mathbf{B}_{21} & \mathbf{B}_{22} & \ldots & \mathbf{B}_{2 N} \\
\vdots & \vdots & \ddots & \vdots \\
\mathbf{B}_{N 1} & \mathbf{B}_{N 2} & \ldots & \mathbf{B}_{N N}
\end{array}\right]
$$

Following exactly the same procedure in [16], we could obtain that $\mathbf{B}_{i j}=\mathbf{0}$ for $i \neq j$ and $\mathbf{B}_{i i}=\mathbf{B}_{j j}$ for $\forall i, j \in\{1, \ldots, N\}$. By defining $\mathbf{B}=\mathbf{B}_{i i}$, we arrive at

$$
\overline{\mathbf{H}}=\mathbf{H B}
$$

from which we can easily show that $\overline{\mathbf{H}}(l)=\mathbf{H}(l) \mathbf{B}$.

From Theorem 1, we know that the dimension of the orthogonal complement space of $\mathcal{K}$ must be $K$. It is also seen that an order over-estimation on each $L_{j, k}$ does not affect the channel identifiability of $\mathbf{H}$ because the estimate $\overline{\mathbf{H}}(l)=\mathbf{H}(l) \mathbf{B}=\mathbf{0}$ for $l=L_{j, k}+1, \ldots, L$ is also correct. Therefore, the two restrictions on the SS method for general MIMO system [12], that is, the requirement of exact channel order and the polynomial matrix ambiguity, are simultaneously lifted in the re-modulated CP-based MIMO OFDM system.

\section{Equalization}

The equalization for CP-based OFDM is quite standard, and we will only bring a brief illustration on this process. Denote the $N$-point DFT of $\mathbf{h}^{(j, k)}$ as

$$
\tilde{\mathbf{h}}^{(j, k)}=\left[\tilde{h}^{(j, k)}(0), \tilde{h}^{(j, k)}(1), \ldots, \tilde{h}^{(j, k)}(N-1)\right]^{T} .
$$

The normalized DFT of $\mathbf{x}_{i}^{(j)}$ has the form

$$
\tilde{\mathbf{x}}_{i}^{(j)}=\left[\tilde{x}_{i}^{(j)}(0), \tilde{x}_{i}^{(j)}(1), \ldots, \tilde{x}_{i}^{(j)}(N-1)\right]^{T}
$$

where

$$
\tilde{x}_{i}^{(j)}(n)=\sum_{k=1}^{K} \tilde{h}^{(j, k)}(n) s_{i}^{(k)}(n)+\zeta_{i}^{(j)}(n), \quad n=0, \ldots, N-1
$$

and $\zeta_{i}^{(j)}(n)$ is the noise after the normalized DFT. For CPbased OFDM, $\zeta_{i}^{(j)}(n)$ is independent Gaussian random variable with respect to pairs $(i, j, n)$ and has the variance $\sigma_{n}^{2}$. This point should be emphasized because it forms one critical difference from ZP-based OFDM, as will be seen later. Let

$$
\begin{aligned}
\tilde{\mathbf{x}}_{i}(n) & =\left[\tilde{x}_{i}^{(1)}(n), \tilde{x}_{i}^{(2)}(n), \ldots, \tilde{x}_{i}^{(J)}(n)\right]^{T} \\
\mathbf{s}_{i}(n) & =\left[s_{i}^{(1)}(n), s_{i}^{(2)}(n), \ldots, s_{i}^{(K)}(n)\right]^{T} \\
\boldsymbol{\zeta}_{i}(n) & =\left[\zeta_{i}^{(1)}(n), \zeta_{i}^{(2)}(n), \ldots, \zeta_{i}^{(J)}(n)\right]^{T} \\
\tilde{\mathbf{H}}(n) & =\left[\begin{array}{ccc}
\tilde{h}^{(1,1)}(n) & \ldots & \tilde{h}^{(1, K)}(n) \\
\vdots & \ddots & \vdots \\
\tilde{h}^{(J, 1)}(n) & \ldots & \tilde{h}^{(J, K)}(n)
\end{array}\right] .
\end{aligned}
$$

Then (43) is expressed as

$$
\tilde{\mathbf{x}}_{i}(n)=\tilde{\mathbf{H}}(n) \mathbf{s}_{i}(n)+\boldsymbol{\zeta}_{i}(n) .
$$

Consequently, the symbol detection could be carried out independently for different carriers. This is one major purpose by using MIMO OFDM systems. That is, the detection could be performed carrier by carrier, which reduces the decoding complexity. Besides, the optimal maximum likelihood (ML) detection could be performed using the efficient sphere decoding (SD) method [19] if $K$ is not large.

\section{E. Comparison with ZPSOS}

1) Similarity: Similarities between these two methods mainly reside in the choice of system parameters and the model structures. For example, under the same transmission rate, namely, the same block length and the $\mathrm{CP}$ length, the channel matrix $\mathcal{G}$ is exactly the same for both methods. The effective SNR, as discussed before, is the same. Similar channel estimation accuracy for both CPSOS and ZPSOS is also observed in the later simulation. Moreover, problems like channel order over-estimation and the identifiability are lifted for both CPSOS and ZPSOS.

2) Difference: Despite many similarities, there exist other differences that show the advantages of CPSOS over ZPSOS.

a) Symbol Detection. In ZP based OFDM, one needs to add the last $L$ entries of $\mathbf{x}_{i}^{(k)}$ to its first $L$ entries before taking the DFT operation. Then, similar relationship as in (45) could be derived for ZPSOS. Note that, $\boldsymbol{\zeta}_{i}(n)$ in ZPSOS, is not independent for different $n$, although its 
entries $\zeta_{i}^{(j)}(n)$ are independent with respect to $j$. Therefore, the ML detection requires the co-consideration of $\tilde{\mathbf{x}}_{i}(n)$ on all carriers. This will cause an exponential increment in the detection complexity, which betrays the original purpose on adopting the MIMO OFDM systems. Although the low complexity Zero Forcing (ZF) detection is suggested in [16], it is well known that this linear detection will cause considerable performance loss.

We here suggest a suboptimal way that the detection still considers each subcarrier independently regardless of whether the noise is dependent across the carriers or not. It can be proved that the covariance matrix of $\zeta_{i}(n)$ in ZPSOS is

$$
\mathrm{E}\left\{\boldsymbol{\zeta}_{i}(n) \boldsymbol{\zeta}_{i}^{H}(n)\right\}=\left(1+\frac{L}{N}\right) \sigma_{n}^{2} \mathbf{I}_{J \times J} .
$$

Therefore, the noise power, compared to CPSOS is increased by a factor of $(1+L / N)$, and the SNR loss is around $10 \log (1+L / N) \mathrm{dB}$. In many standards, e.g. IEEE 802.11a, IEEE 802.11n, $N=4 L$ is adopted and the SNR loss is around $1 \mathrm{~dB}$.

b) Compatibility. Obviously, the CP-based OFDM has a much wider application than the ZP-based OFDM. For example, CP-based OFDM has been well adopted into European digital audio/video broadcasting (DAB, DVB) [20], [21], high performance local area network (HIPERLAN) [22], IEEE 802.11a WLAN standards and the coming IEEE 802.11n WLAN standards. However, to the best of authors' knowledge, the ZP based transmission has very limited applications.

\section{Asymptotical Performance AnAlysis}

\section{A. Channel Estimation Mean Square Error}

We provide a first-order performance analysis on the proposed estimator at high SNR similar to that adopted for DSCDMA in [23], [24].

Theorem 2: Assume that both noise and signals are zeromean i.i.d. with variances $\sigma_{n}^{2}$ and $\sigma_{s}^{2}$, respectively, the covariance of the channel estimation error is approximated by

$$
\mathrm{E}\left\{\operatorname{vec}(\Delta \mathbf{H}) \operatorname{vec}^{H}(\Delta \mathbf{H})\right\}=\mathbf{I}_{K} \otimes\left(\frac{\sigma_{n}^{2}\left(\mathcal{K}^{H}\right)^{\dagger} \mathcal{K}^{\dagger}}{2 M \sigma_{s}^{2}}\right) .
$$

Specifically, the error covariance matrix for the $k$ th transmit antenna is

$$
\mathrm{E}\left\{\Delta \mathbf{H}(:, k) \Delta \mathbf{H}^{H}(:, k)\right\}=\frac{\sigma_{n}^{2}\left(\mathcal{K}^{H}\right)^{\dagger} \mathcal{K}^{\dagger}}{2 M \sigma_{s}^{2}}
$$

and the channel estimation MSE is

$$
\mathrm{E}\left\{\|\Delta \mathbf{H}(:, k)\|^{2}\right\}=\frac{\sigma_{n}^{2}\left\|\mathcal{K}^{\dagger}\right\|_{F}^{2}}{2 M \sigma_{s}^{2}} .
$$

See proof in Appendix I. Several insightful observations can be drawn from (48), for example, the MSE is proportional to the noise power but is inversely proportional to both the signal power and the number of the received signal block.

\section{B. Cramér-Rao-Bound (CRB)}

The absolute CRB should be obtained from the most original equation (9). However, since the performance of the provided algorithm is only related (12), we would like to resort to the $\mathrm{CRB}$ after the pre-processing of our re-modulation. By using this CRB, we can also build up its relationship with the MSE that is derived in the previous subsection.

The deterministic CRB [25] for CPSOS will be considered here, where the observations are $\mathbf{z}=\left[\mathbf{z}_{1}^{T}, \ldots, \mathbf{z}_{M}^{T}\right]^{T}$, and the parameters to be estimated are $\boldsymbol{\theta}=\left[\operatorname{vec}(\mathbf{H}), \mathbf{d}_{1}, \ldots, \mathbf{d}_{M}, \sigma_{n}^{2}\right]$. To calculate CRB, we need the joint probability density function (PDF) of $\mathbf{z}$, denoted as $p(\mathbf{z} \mid \boldsymbol{\theta})$. Since $\boldsymbol{\eta}_{i}$ is correlated with both $\boldsymbol{\eta}_{i-1}$ and $\boldsymbol{\eta}_{i+1}$, the covariance matrix of $\mathbf{z}$, denoted as $\mathbf{R}_{z}$, is an $M J(N+L) \times M J(N+L)$ Toeplitz matrix with the main diagonal elements 2 , the $(J N+1)$ th, $-(J N+1)$ th $^{2}$ diagonal elements -1 , and all other elements 0 . We note that the inverse of such a huge Toeplitz matrix is mathematically prohibitive.

To simplify the derivation and gain more insight into the proposed algorithm, we approximate $\mathbf{R}_{z}$ by

$$
\mathbf{R}_{z}=\mathbf{I}_{M} \otimes \mathbf{R}_{w}
$$

which equivalently says that we ignore the correlations among different $\boldsymbol{\eta}_{i}$ 's. This approximation is also justified since the performance of SS method is only related to the autocovariance of $\boldsymbol{\eta}_{i}$ and does not depend on whether $\boldsymbol{\eta}_{i}$ are cross-correlated or not. The so derived CRB will be called as approximated $\mathrm{CRB}$ (ACRB). Since we relax the noise condition, ACRB should be greater than or equal to the CRB.

Define

$$
\mathcal{D}_{i}=\left[\mathbf{D}_{i}^{(1)}, \mathbf{D}_{i}^{(2)}, \ldots, \mathbf{D}_{i}^{(K)}\right]
$$

where

$$
\mathbf{D}_{i}^{(k)}=\sum_{n=1}^{N} \mathbf{C}_{n} d_{i}((n-1) K+k) .
$$

The ACRB is obtained as

$$
\operatorname{ACRB}_{\mathrm{vec}(\mathbf{H})}=\sigma_{n}^{2}\left(\sum_{i=1}^{M} \mathcal{D}_{i}^{H} \mathbf{R}_{w}^{-1 / 2} \mathbf{P}_{\mathcal{A}}^{\perp} \mathbf{R}_{w}^{-1 / 2} \mathcal{D}_{i}\right)^{\dagger}
$$

where $\mathbf{P}_{\mathcal{A}}^{\perp}$ is the projection matrix onto the orthogonal complement space spanned by $\mathcal{A}^{\perp}$. If signals are i.i.d. with variance $\sigma_{s}^{2}$, the asymptotical ACRB for large $M$ is obtained as

$$
\mathrm{ACRB}_{\mathrm{vec}(\mathbf{H})}=\mathbf{I}_{K} \otimes\left(\frac{\sigma_{n}^{2}\left(\mathcal{K}^{H}\right)^{\dagger} \mathcal{K}^{\dagger}}{2 M \sigma_{s}^{2}}\right)
$$

and the asymptotical ACRB for each column of $\mathbf{H}$ can be separately, obtained as

$$
\mathrm{ACRB}_{\operatorname{vec}(\mathbf{H}(:, k))}=\frac{\sigma_{n}^{2}\left(\mathcal{K}^{H}\right)^{\dagger} \mathcal{K}^{\dagger}}{2 M \sigma_{s}^{2}} .
$$

See proof in Appendix II.

Interestingly, the asymptotical ACRB is the same as the asymptotical error covariance matrix (48). Nonetheless, the channel estimation MSE is greater than or equal to the CRB, which agrees with the intuition very well. 


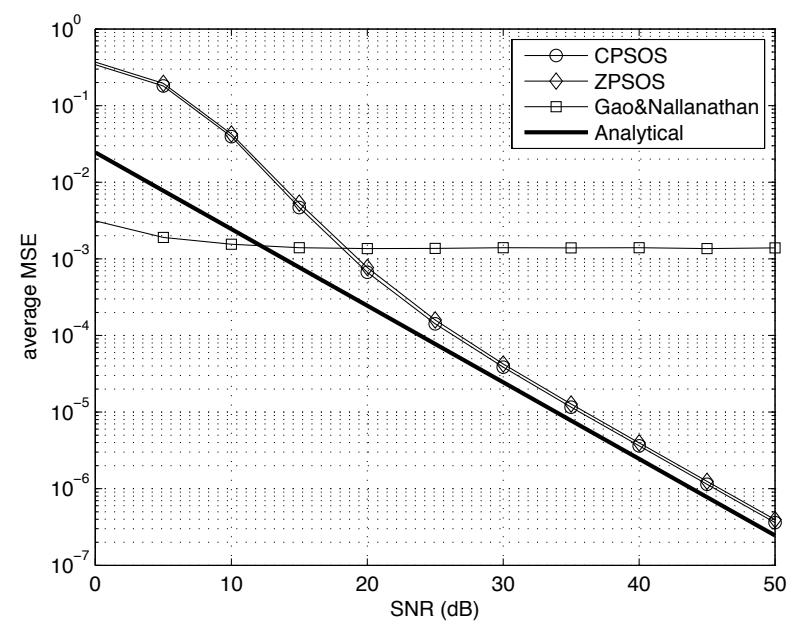

Fig. 2. Channel estimation MSE versus SNR with 200 received blocks.

\section{Simulation Results}

In this section, we examine the performance of CPSOS for a $2 \times 2$ MIMO OFDM systems under various scenarios. The OFDM block length is taken as $N=32$, and the CP length is taken as $L=8$. The symbols are extracted from Quadratic Phase Shift Keying (QPSK) constellations. The 6-ray channel model with an exponential power delay profile

$$
\mathrm{E}\left\{\left|h^{(j, k)}(l)\right|^{2}\right\}=\rho \exp (-l / 5), \quad l=0, \ldots, 5
$$

is used where $\rho$ is the coefficient to normalize the overall channel gain to $\left\|\mathbf{h}^{(j, k)}\right\|^{2}=1$. The estimation MSE is defined as

$$
\mathrm{MSE}=\frac{1}{J K}\left\|\hat{\mathbf{H}} \mathrm{B}^{-1}-\mathbf{H}\right\|_{F}^{2}
$$

where, for simulation purpose, the ambiguity matrix $\mathbf{B}$ is obtained according to [26]:

$$
\mathbf{B}=\arg \min _{\mathbf{B}}\left\|\hat{\mathbf{H}} \mathbf{B}^{-1}-\mathbf{H}\right\|_{F}^{2} .
$$

The number of the Monte-Carlo runs used for average is taken as 500 .

We first fix the number of the OFDM blocks as 200 and compare three different blind channel estimators: CPSOS, ZPSOS and Gao\&Nallanathan's method [17]. For Gao\&Nallanathan's method, the value of $p$ is chosen as 0.5 . Note that 200 blocks is a common number for applying the SS algorithm. The channel estimation MSE versus SNR for these three algorithms are shown in Fig. 2. The analytical performance derived from either asymptotical MSE or ACRB is displayed as well. It is seen that ZPSOS and CPSOS give comparable performance over all SNR considered. Both methods are close to the analytical MSE after $20 \mathrm{~dB}$. The gap between the analytical result and the simulation result is due to the asymptotical MSE being used here. One may expect a little bit better performance from CPSOS because the noise is colored with a known covariance matrix. On the other hand, Gao\&Nallanathan's method gives better performance at lower SNR region but keeps constant when SNR is greater than

\footnotetext{
${ }^{2}$ Notations follow those in MATLAB.
}

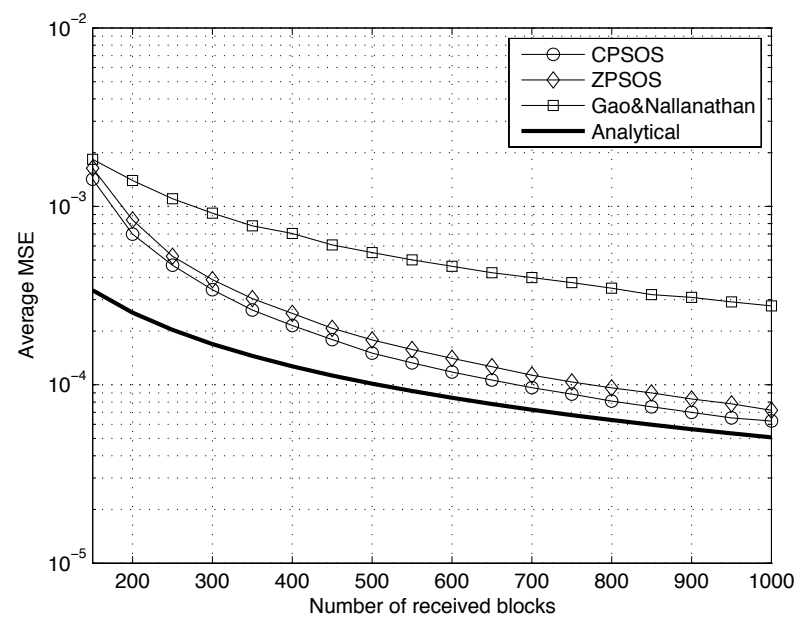

Fig. 3. Channel estimation MSEs versus number of OFDM blocks for $\mathrm{SNR}=$ $20 \mathrm{~dB}$.

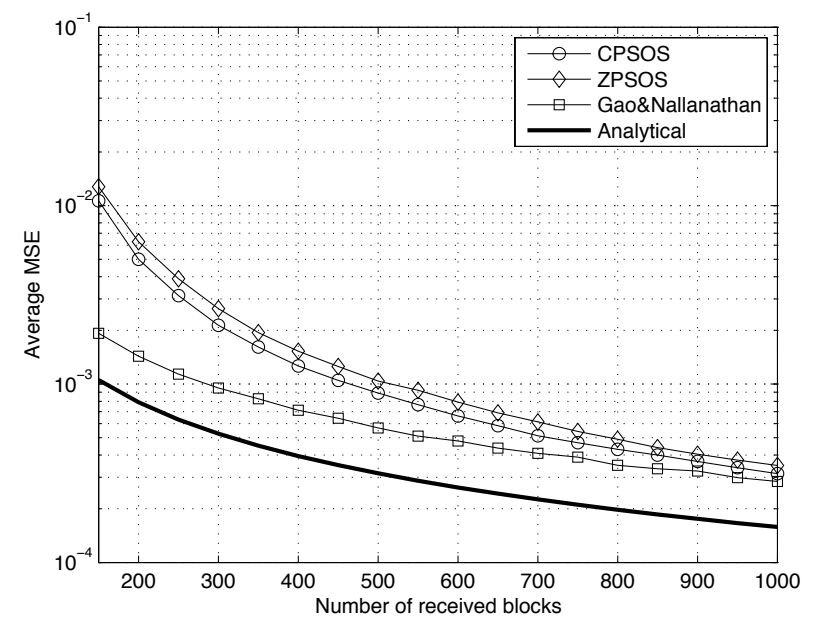

Fig. 4. Channel estimation MSEs versus number of OFDM blocks for SNR= $15 \mathrm{~dB}$.

$10 \mathrm{~dB}$. We should mention that, the proposed ACRB is not applicable to Gao\&Nallanathan's method because the method is based on a different signal model.

Fig. 3 and Fig. 4 show the performance of MSE versus the number of OFDM blocks for the three algorithms at $\mathrm{SNR}=20 \mathrm{~dB}$ and $\mathrm{SNR}=15 \mathrm{~dB}$, respectively. As explained previously, the performance of CPSOS is a little bit better than ZPSOS and it may achieve the analytical asymptotical MSE when the number of blocks becomes large. Although Gao\&Nallanathan's method does not meet any error floor with the increase of number of blocks, it is outperformed by CPSOS over all block region at $\mathrm{SNR}=20 \mathrm{~dB}$. It is also known from Fig. 4 that the improvement of Gao\&Nallanathan's method with the increment of number of blocks is slower than that of CPSOS. Therefore, we may expect that CPSOS can asymptotically outperform Gao\&Nallanathan's method for sufficiently large number of blocks at any SNR value.

Fig. 5 and Fig. 6 show the amplitude of the channel tap detection of four random channel realizations at $\mathrm{SNR}=12 \mathrm{~dB}$ and $\mathrm{SNR}=20 \mathrm{~dB}$, respectively. It is noted that $\mathrm{SNR}=12 \mathrm{~dB}$ 


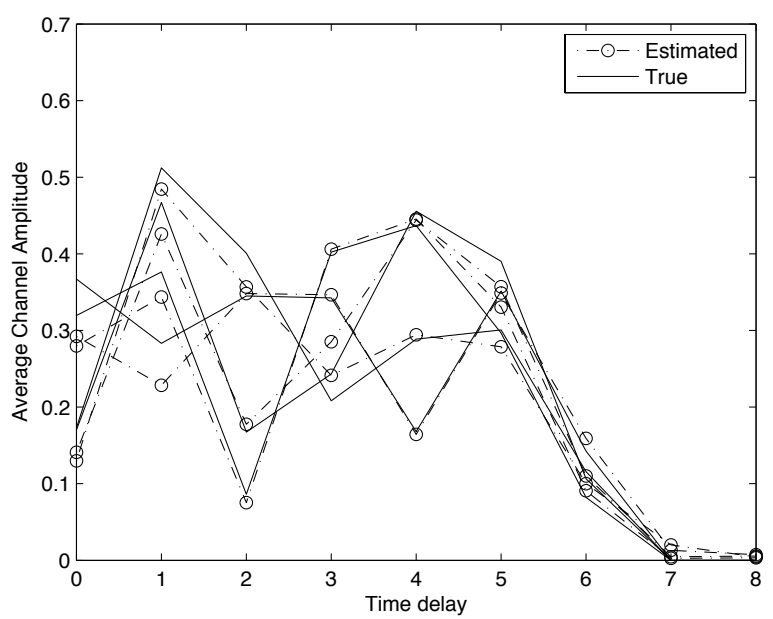

Fig. 5. Amplitude estimation of channel taps at $\mathrm{SNR}=12 \mathrm{~dB}$.

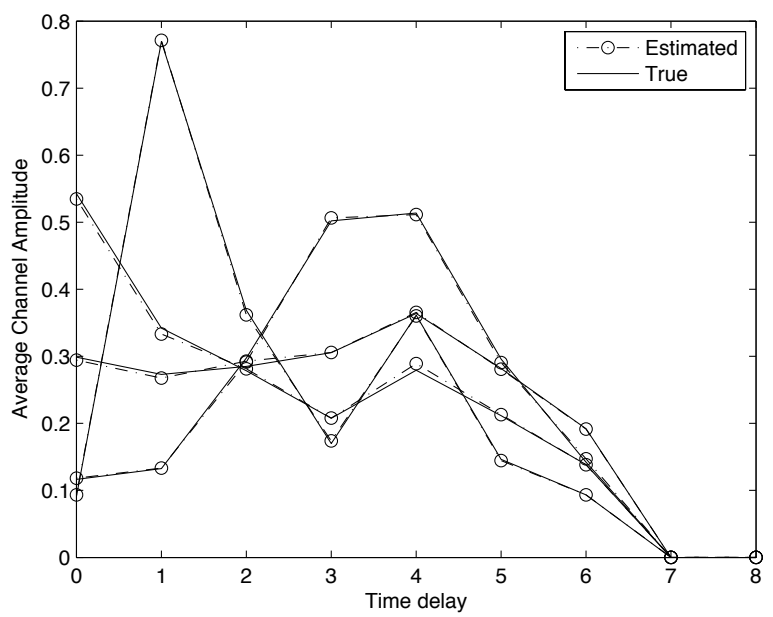

Fig. 6. Amplitude estimation of channel taps at $\mathrm{SNR}=20 \mathrm{~dB}$.

yields relatively good channel amplitude estimation, whereas $\mathrm{SNR}=20 \mathrm{~dB}$ gives almost perfect estimation. Nevertheless, by noting that the channel amplitude at tap 7 and tap 8 are very near to zero in both figures, we may also apply the channel order estimation method suggested in [16] to the proposed CPSOS.

To demonstrate the robustness of CPSOS to the channel order over-estimation, we assume the estimated channel order as $\hat{L}=5,6,7$ respectively. The value $\hat{L}=5$ corresponds to the correct channel order, and other values are those being overestimated. The channel estimation MSE versus SNR and block number for these three different orders are displayed in Fig. 7 and Fig. 8, respectively. As expected, order over-estimation only causes slight performance loss. This is reasonable that assuming more channel taps, even if those zero taps, may also contribute to the channel estimation error. Nevertheless, the largest loss, appearing when the order is taken the same as the $\mathrm{CP}$ length, is less than $1 \mathrm{~dB}$.

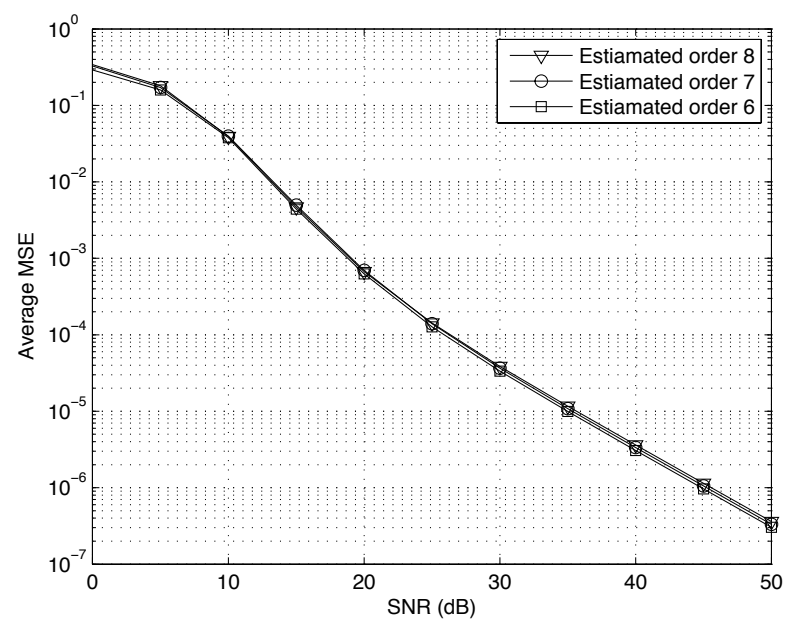

Fig. 7. Channel estimation MSEs versus SNR for different estimated channel order.

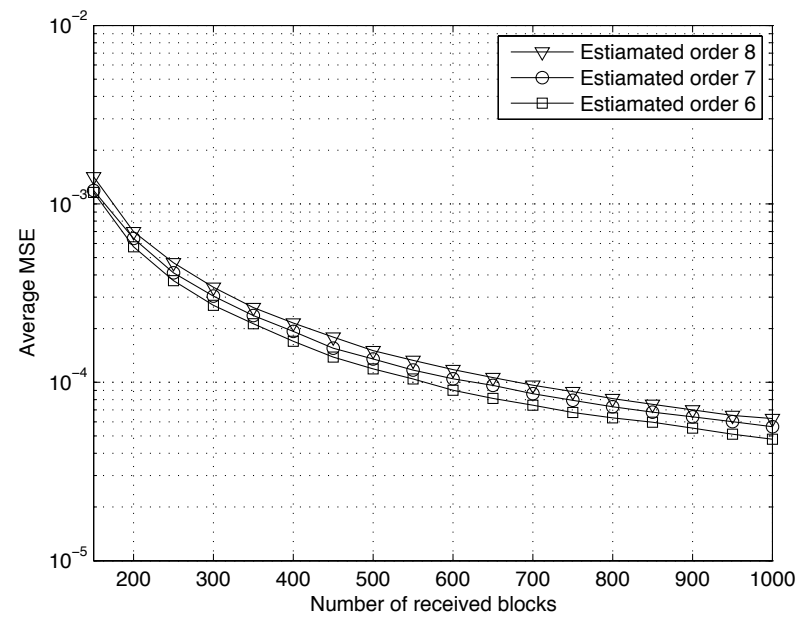

Fig. 8. Channel estimation MSEs versus number of OFDM blocks for different estimated channel order.

\section{CONCLUSIONS}

In this paper, we have developed a new SS based blind channel estimation for MIMO OFDM systems. With an appropriate re-modulation on the received signals, an effective way has been found to apply the SS method for the CP based MIMO-OFDM system when the number of receiver antennas is no less than the number of transmitter antennas. Most issues related to the SS method have been studied for this newly proposed modulation, e.g. channel identifiability, order over-estimation, MSE of the channel estimation as well as the CRB of the channel estimation. Most importantly, since the proposed method allows blind channel estimation for the CP based MIMO OFDM, it is compatible with many existing standards and the coming $4 \mathrm{G}$ wireless communication standards.

\section{APPENDIX A}

\section{Derivation of The ChANNEl Estimation MSE}

Firstly, we introduce the lemma provided in [27]. 
Lemma 2 [27]: Denote the singular value decomposition (SVD) of

$$
\mathbf{Y}=\mathcal{A}\left[\mathbf{d}_{1}, \ldots, \mathbf{d}_{M}\right]=\mathcal{A D}
$$

as

$$
\mathbf{Y}=\left[\begin{array}{ll}
\mathbf{U}_{s} & \mathbf{U}_{o}
\end{array}\right]\left[\begin{array}{cc}
\boldsymbol{\Sigma}_{s} & \mathbf{0} \\
\mathbf{0} & \mathbf{0}
\end{array}\right]\left[\begin{array}{l}
\mathbf{V}_{s}^{H} \\
\mathbf{V}_{o}^{H}
\end{array}\right]
$$

The first order approximation of the perturbation to $\mathbf{U}_{o}$ due to the additive noise $\mathbf{N}=\left[\tilde{\mathbf{n}}_{1}, \ldots, \tilde{\mathbf{n}}_{M}\right]$ is

$$
\Delta \mathbf{U}_{o}=-\mathbf{U}_{s} \boldsymbol{\Sigma}_{s}^{-1} \mathbf{V}_{s}^{H} \mathbf{N}^{H} \mathbf{U}_{o}=-\left(\mathbf{Y}^{H}\right)^{\dagger} \mathbf{N}^{H} \mathbf{U}_{o} .
$$

Ideally, the channel matrix $\mathbf{H}$ is obtained from

$$
\mathcal{K}^{H} \mathbf{H}=\mathbf{0} .
$$

However, we may only be able to obtain an orthonormal matrix $\hat{\mathbf{H}}$ from the left singular vectors of $\mathcal{K}$. Therefore, $\mathbf{H}$ is expressed as $\mathbf{H}=\hat{\mathbf{H}} \mathbf{B}^{-1}$ for an unknown $\mathbf{B}$. By applying Lemma 2 again, the perturbation of the channel estimate $\hat{\mathbf{H}}$ is

$$
\Delta \hat{\mathbf{H}}=-\left(\mathcal{K}^{H}\right)^{\dagger} \Delta \mathcal{K}^{H} \hat{\mathbf{H}}
$$

where

$$
\begin{aligned}
& \Delta \mathcal{K}=\left[\mathbf{C}_{1}^{H} \mathbf{R}_{w}^{-1 / 2} \Delta \mathbf{U}_{o}, \ldots, \mathbf{C}_{N}^{H} \mathbf{R}_{w}^{-1 / 2} \Delta \mathbf{U}_{o}\right] \\
&=-\left[\mathbf{C}_{1}^{H} \mathbf{R}_{w}^{-1 / 2}\left(\mathbf{Y}^{H}\right)^{\dagger} \mathbf{N}^{H} \mathbf{U}_{o}, \ldots,\right. \\
&\left.\mathbf{C}_{N}^{H} \mathbf{R}_{w}^{-1 / 2}\left(\mathbf{Y}^{H}\right)^{\dagger} \mathbf{N}^{H} \mathbf{U}_{o}\right] .
\end{aligned}
$$

It then follows

$$
\begin{aligned}
\Delta \mathbf{H} & =\Delta \hat{\mathbf{H}} \mathbf{B}^{-1}=-\left(\mathcal{K}^{H}\right)^{\dagger} \Delta \mathcal{K}^{H} \hat{\mathbf{H}} \mathbf{B}^{-1} \\
& =-\left(\mathcal{K}^{H}\right)^{\dagger} \Delta \mathcal{K}^{H} \mathbf{H} .
\end{aligned}
$$

Note that (65) could not be directly derived from Lemma 2 since Lemma 2 is only applicable for perturbation in the eigenspace. From [24], we know that $\mathrm{E}\left\{\mathbf{N Q N}^{H}\right\}=\sigma_{n}^{2} \operatorname{tr}(\mathbf{Q}) \mathbf{I}$. Therefore,

$$
\begin{aligned}
& \mathrm{E}\left\{\mathbf{N} \mathbf{Y}^{\dagger} \mathbf{R}_{w}^{-1 / 2} \mathbf{C}_{m} \mathbf{H}(:, k) \mathbf{H}^{H}(:, p) \mathbf{C}_{n}^{H} \mathbf{R}_{w}^{-1 / 2}\left(\mathbf{Y}^{H}\right)^{\dagger} \mathbf{N}^{H}\right\} \\
= & \sigma_{n}^{2} \operatorname{tr}\left(\mathbf{Y}^{\dagger} \mathbf{R}_{w}^{-1 / 2} \mathbf{C}_{m} \mathbf{H}(:, k) \mathbf{H}^{H}(:, p) \mathbf{C}_{n}^{H} \mathbf{R}_{w}^{-1 / 2}\left(\mathbf{Y}^{H}\right)^{\dagger}\right) \mathbf{I} \\
= & \sigma_{n}^{2} \operatorname{tr}\left(\mathcal{A}_{n, p}^{H}\left(\mathbf{Y}^{H}\right)^{\dagger} \mathbf{Y}^{\dagger} \mathcal{A}_{m, k}\right) \mathbf{I} \\
= & \sigma_{n}^{2} \operatorname{tr}\left(\mathcal{A}_{n, p}^{H}\left(\mathbf{Y} \mathbf{Y}^{H}\right)^{\dagger} \mathcal{A}_{m, k}\right) \mathbf{I} \\
= & \sigma_{n}^{2} \operatorname{tr}\left(\mathcal{A}_{n, p}^{H}\left(\mathcal{A}^{H}\right)^{\dagger}\left(\mathbf{D D}^{H}\right)^{-1} \mathcal{A}^{\dagger} \mathcal{A}_{m, k}\right) \mathbf{I} \\
= & \sigma_{n}^{2} \operatorname{tr}\left(\mathbf{e}_{(n-1) K+p}^{H}\left(\mathbf{D D}^{H}\right)^{-1} \mathbf{e}_{(m-1) K+k}\right) \mathbf{I}
\end{aligned}
$$

where $\mathcal{A}_{m, k}$ is the $((m-1) K+k)$ th column of $\mathcal{A}$, and the property $\left(\mathbf{Y} \mathbf{Y}^{H}\right)^{\dagger}=\left(\mathbf{Y}^{H}\right)^{\dagger} \mathbf{Y}^{\dagger}$ is used. The term $\mathbf{D} \mathbf{D}^{H}$ is in fact the estimated signal covariance matrix $2 \sigma_{s}^{2} M \mathbf{I}$ for the asymptotically large $M$. Therefore, equation (66) can be well approximated by

$$
\begin{aligned}
& \sigma_{n}^{2} \operatorname{tr}\left(\mathbf{e}_{(n-1) K+p}^{H}\left(\mathbf{D D}^{H}\right)^{-1} \mathbf{e}_{(m-1) K+k}\right) \mathbf{I} \\
= & \frac{\sigma_{n}^{2}}{2 M \sigma_{s}^{2}} \delta_{m-n} \delta_{p-k} \mathbf{I} .
\end{aligned}
$$

Finally, the channel error covariance matrix can be obtained as

$$
\begin{aligned}
& \mathrm{E}\left\{\operatorname{vec}(\Delta \mathbf{H}) \operatorname{vec}^{H}(\Delta \mathbf{H})\right\} \\
= & \left(\mathcal{K}^{H}\right)^{\dagger} \operatorname{E}\left\{\Delta \mathcal{K}^{H} \operatorname{vec}(\mathbf{H}) \operatorname{vec}^{H}(\mathbf{H}) \Delta \mathcal{K}\right\} \mathcal{K}^{\dagger} \\
= & \mathbf{I}_{K} \otimes\left(\frac{\sigma_{n}^{2}}{2 M \sigma_{s}^{2}}\left(\mathcal{K}^{H}\right)^{\dagger} \mathbf{U}_{o}^{H} \mathbf{U}_{o} \mathcal{K}^{\dagger}\right) \\
= & \mathbf{I}_{K} \otimes\left(\frac{\sigma_{n}^{2}\left(\mathcal{K}^{H}\right)^{\dagger} \mathcal{K}^{\dagger}}{2 M \sigma_{s}^{2}}\right) .
\end{aligned}
$$

\section{APPENDIX B}

\section{DERIVATION OF THE CRB}

From the approximation of (50), it is suffice to first consider $\mathbf{z}_{i}$, and the unknown parameters changes to $\boldsymbol{\theta}=$ $\left[\operatorname{vec}(\mathbf{H}), \mathbf{d}_{i}, \sigma_{n}^{2}\right]$. The exact FIM for $\boldsymbol{\vartheta}=\left[\operatorname{vec}(\mathbf{H}), \mathbf{d}_{i}\right]$ can be expressed from [25]

$$
\mathbf{J}=\frac{1}{\sigma_{n}^{2}} \Gamma^{H} \mathbf{R}_{w}^{-1} \boldsymbol{\Gamma}
$$

where

$$
\boldsymbol{\Gamma}=\left[\frac{\partial\left(\mathcal{A} \mathbf{d}_{i}\right)}{\partial \operatorname{vec}(\mathbf{H})}, \frac{\partial\left(\mathcal{A} \mathbf{d}_{i}\right)}{\partial \mathbf{d}_{i}}\right] .
$$

It can be obtained straight-forwardly that

$$
\begin{aligned}
& \frac{\partial\left(\mathcal{A} \mathbf{d}_{i}\right)}{\partial \operatorname{vec}(\mathbf{H})}=\mathbf{R}_{w}^{-1 / 2} \mathcal{D}_{i}, \\
& \frac{\partial\left(\mathcal{A} \mathbf{d}_{i}\right)}{\partial \mathbf{d}_{i}}=\mathcal{A} .
\end{aligned}
$$

From [25], we know that for blind channel estimation, the FIM is singular such that its inverse does not exist. Then, some constraints should be utilized to make $\mathbf{J}$ a non-singular matrix. Instead of taking any specific constraint, we use the minimal constrained CRB defined as in [25].

Lemma 3 [25]: Suppose the FIM for $\boldsymbol{\vartheta}=\left[\operatorname{vec}(\mathbf{H}), \mathbf{d}_{i}\right]^{T}$ is

$$
\mathbf{J}=\frac{1}{\sigma_{n}^{2}}\left[\begin{array}{ll}
\mathbf{J}_{11} & \mathbf{J}_{12} \\
\mathbf{J}_{21} & \mathbf{J}_{22}
\end{array}\right],
$$

where $\mathbf{J}_{11}$ is of dimension $K J(L+1) \times K J(L+1)$ and assume $\mathbf{J}$ is singular but $\mathbf{J}_{22}$ is nonsingular. Then, the minimal constrained CRB for $\operatorname{vec}(\mathbf{H})$ is

$$
\mathrm{CRB}_{\mathrm{vec}(\mathbf{H})}=\sigma_{n}^{2}\left[\mathbf{J}_{11}-\mathbf{J}_{12} \mathbf{J}_{22}^{-1} \mathbf{J}_{21}\right]^{\dagger} .
$$

This is a particular constrained CRB that yields the lowest value for $\operatorname{tr}\{\mathrm{CRB}\}$ among all lists of a minimal number of independent constraints.

Applying the above lemma, we obtain

$$
\begin{aligned}
& \mathrm{ACRB}_{\mathrm{vec}(\mathbf{H})} \\
= & \sigma_{n}^{2}\left(\mathcal{D}_{i}^{H} \mathbf{R}_{w}^{-1} \mathcal{D}-\mathcal{D}^{H} \mathbf{R}_{w}^{-1 / 2} \mathcal{A}\left(\mathcal{A}^{H} \mathcal{A}\right)^{-1} \mathcal{A}^{H} \mathbf{R}_{w}^{-1 / 2} \mathbf{D}_{i}\right)^{\dagger} \\
= & \sigma_{n}^{2}\left(\mathcal{D}_{i}^{H} \mathbf{R}_{w}^{-1 / 2}\left(\mathbf{I}-\mathcal{A}\left(\mathcal{A}^{H} \mathcal{A} \mathcal{A}^{H}\right)^{-1}\right) \mathbf{R}_{w}^{-1 / 2} \mathcal{D}_{i}\right)^{\dagger} \\
= & \sigma_{n}^{2}\left(\mathcal{D}_{i}^{H} \mathbf{R}_{w}^{-1 / 2} \mathbf{P}_{\mathcal{A}}^{\perp} \mathbf{R}_{w}^{-1 / 2} \mathcal{D}_{i}\right)^{\dagger} \\
= & \sigma_{n}^{2}\left(\mathcal{D}_{i}^{H} \mathbf{R}_{w}^{-1 / 2} \mathbf{U}_{o} \mathbf{U}_{o}^{H} \mathbf{R}_{w}^{-1 / 2} \mathcal{D}_{i}\right)^{\dagger}
\end{aligned}
$$

From the approximation, the noise $\eta_{i}$ can be considered independent for each $i$. Then, the ACRB, by observing $\mathbf{z}$, can be found directly from

$$
\operatorname{ACRB}_{\operatorname{vec}(\mathbf{H})}=\sigma_{n}^{2}\left(\sum_{i=1}^{M} \mathcal{D}_{i}^{H} \mathbf{R}_{w}^{-1 / 2} \mathbf{U}_{o} \mathbf{U}_{o}^{H} \mathbf{R}_{w}^{-1 / 2} \mathcal{D}_{i}\right)^{\dagger} .
$$


Asymptotically, we have

$$
\begin{aligned}
& \sum_{i=1}^{M}\left(\mathbf{D}_{i}^{(k)}\right)^{H} \mathbf{R}_{w}^{-1 / 2} \mathbf{U}_{o} \mathbf{U}_{o}^{H} \mathbf{R}_{w}^{-1 / 2} \mathbf{D}_{i}^{(p)} \\
= & \sum_{n=1}^{N} \sum_{m=1}^{N} \mathbf{C}_{n}^{H} \mathbf{R}_{w}^{-1 / 2} \mathbf{U}_{o} \mathbf{U}_{o}^{H} \mathbf{R}_{w}^{-1 / 2} \mathbf{C}_{m} \\
& \times \sum_{i=1}^{M} d_{i}^{*}((n-1) K+k) d_{i}((m-1) K+p) \\
\approx & 2 M \sigma_{s}^{2} \sum_{n=1}^{N} \mathbf{C}_{n}^{H} \mathbf{R}_{w}^{-1 / 2} \mathbf{U}_{o} \mathbf{U}_{o}^{H} \mathbf{R}_{w}^{-1 / 2} \mathbf{C}_{n} \delta_{k-p} \\
= & 2 M \sigma_{s}^{2} \mathcal{K} \mathcal{K}^{H} \delta_{k-p} .
\end{aligned}
$$

Equation (77) is obtained asymptotically for large $M$, bearing in mind that elements of $\mathbf{d}_{i}$ are i.i.d. with variance $2 \sigma_{s}^{2}$ if $s_{i}^{(k)}(n)$ are i.i.d. with variance $\sigma_{s}^{2}$. Therefore, the ACRB for $\operatorname{vec}(\mathbf{H})$ is

$$
\begin{aligned}
\operatorname{ACRB}_{\mathrm{vec}(\mathbf{H})} & =\sigma_{n}^{2}\left(\mathbf{I}_{K} \otimes\left(2 M \sigma_{s}^{2} \mathcal{K K}^{H}\right)\right)^{\dagger} \\
& =\frac{\sigma_{n}^{2}}{2 M \sigma_{s}^{2}} \mathbf{I}_{K} \otimes\left(\mathcal{K} \mathcal{K}^{H}\right)^{\dagger} \\
& =\mathbf{I}_{K} \otimes\left(\frac{\sigma_{n}^{2}\left(\mathcal{K}^{H}\right)^{\dagger} \mathcal{K}^{\dagger}}{2 M \sigma_{s}^{2}}\right)
\end{aligned}
$$

\section{REFERENCES}

[1] J. Bingham, "Multicarrier modulation for data transmission: an idea whose time has come," IEEE Commun. Mag., vol. 28, pp. 5-14, May 1990.

[2] I. E. Telatar, "Capacity of multi-antenna Gaussian channels," Bell Labs Technical Memorandum, 1995.

[3] G. J. Foschini, and M. J. Gans, "On limits of wireless communications in a fading environment when using multiple antennas," Wireless Personal Commun., vol. 6, pp. 311-335, 1998.

[4] H. Sampath, S. Talwar, J. Tellado, V. Erceg, and A. Paulraj, "A fourth-generation MIMO-OFDM broadband wireless system: design, performance, and field trial results," IEEE Commun. Mag., pp. 143-149, Sept. 2002.

[5] Y. Li, N. Seshadri, and S. Ariyavisitakul, "Channel estimation for OFDM systems with transmitter diversity in mobile wireless channels", IEEE J. Select. Areas Commun., vol. 17, pp. 461C471, Mar. 1999.

[6] I. Barhumi, G. Leus, and M. Moonen, "Optimal training design for MIMO OFDM systems in mobile wireless channels," IEEE Trans. Signal Processing, vol. 51, pp. 1615-1624, June, 2003.

[7] H. Minn, and N. Al-Dhahir, "Optimal training signals for MIMO OFDM channel estimation," IEEE Trans. Wireless Commun., vol. 5, pp. 11581168, May 2006.

[8] L. Tong, and S. Perreau, "Multichannel blind identification: from subspace to maximum likelihood methods," in Proc. IEEE, vol. 86, pp. 1951-1968, Oct. 1998.

[9] E. Moulines, P. Duhamel, J. F. Cardoso, and S. Mayrargue, "Subspace methods for the blind identification of multichannel FIR filters," IEEE Trans. Signal Processing, vol. 43, pp. 516-525, Feb. 1995.

[10] E. de Carvalho, and D. Slock, "Blind and semi-blind FIR multichannel estimation: (global) identifiability conditions," IEEE Trans. Signal Processing, vol. 52, pp. 1053-1064, Apr. 2004.

[11] A. Medles and D. Slock, "Linear precoding for spatial multiplexing MIMO systems: blind channel estimation aspects," in Proc. IEEE ICC'02, New York, USA, vol. 1, pp. 401-405, 28 April-2 May 2002.

[12] K. Abed-Meraim, P. Loubaton, and E. Moulines, "A subspace algorithm for certain blind identification problem," IEEE Trans. Inform. Theory, vol. 32, pp. 499-511, Apr. 1997.

[13] J. Lorincz, and D. Begusic, "Physical layer analysis of emerging IEEE 802.11n WLAN standard," Proc. ICACT' 06, Phoenix Park, Korea, vol. 1, pp. 189-194, Feb. 2006.

[14] TGn Sync Proposal Technical Specification IEEE 802.11-05/1095r2.
[15] "Wireless LAN medium access control (MAC) and physical layer (PHY) specifications: high speed physical layer in the 5 GHZ band," IEEE802.11a, 1999.

[16] Y. Zeng, and T. S. Ng, "A semi-blind channel estimation method for multiuser multiantenna OFDM systems," IEEE Trans. Signal Processing, vol. 52, pp. 1419-1429, May, 2004

[17] F. Gao, and A. Nallanathan, "Blind Channel Estimation for MIMO OFDM Systems via Nonredundant Linear Precoding," IEEE Trans. Signal Processing, vol. 55, pp. 784-789, Jan. 2007.

[18] A. Gorokhov and P. Loubaton, "Subspace-based techniques for blind separation of convolutive mixtures with temporally correlated sources," IEEE Trans. Circuits Syst. I, vol. 44, pp. 813-820, Sept. 1997.

[19] E. Viterbo and J. Boutros, "A universal lattice code decoder for fading channels," IEEE Trans Inform. Theory, vol. 45, pp. 1639-1642, July 1999.

[20] "Radio broadcasing system, digital audio broadcasting (DAB) to mobile, portable, and fixed receiver," Eur. Telecommun. Stand. Inst., SophiaAntipolis, Valbonne, France, ETS 300 401, 1995-1997.

[21] "Digital broadcasting system television, sound, and data services; framing structure, channel coding, and modulation digital terrestrial television," Eur. Telecommun. Stand. Inst., Sophia-Antipolis, Valbonne, France, ETS 300 744, 1996.

[22] "Broadband radio access networks (BRAN): high performance radio local area networks (HIPERLAN), type 2; Systems overview," Eur. Telecommun. Stand. Inst., Sophia-Antipolis, Valbonne, France, ETR 101 $683114,1999$.

[23] H. Liu, and G. Xu, "A subspace method for signature waveform estimation in synchronous CDMA systems," IEEE Trans. Commun., vol. 44, pp. 1346-1354, Oct. 1996.

[24] S. Roy, and C. Li, " A subspace blind channel estimation method for OFDM systems without cyclic prefix," IEEE Trans. Wireless Commun., vol. 1, pp. 572-579, Oct. 2002.

[25] E. de Carvalho, J. Cioffi, and D. Slock, "Cramér-rao bounds for blind multichannel estimation," in Proc. IEEE GLOBECOM'00, San Francisco, USA, Nov. 2000, vol. 2, pp. 1036-1040.

[26] H. Gazzah, P. A. Regalia, J. P. Delmas, and K. Abed-Meraim, "A blind multichannel identification algorithm robust to order overestimation," IEEE Trans. Signal Processing, vol. 50, pp. 1449-1458, June 2002.

[27] F. Li, H. Liu, and R. J. Vaccaro, "Performance analysis for DOA estimation algorithms: futher unification, simplification, and observations," IEEE Trans. Aerosp., Electron. Syst., vol. 29, pp. 1170-1184, Oct. 1993.

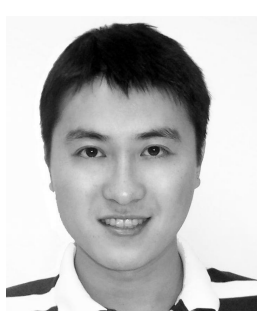

Feifei Gao (S'05) received the B.Eng. degree in information engineering from Xi'an Jiaotong University, Xi'an, Shaanxi China, in 2002, the M.Sc. degree from the McMaster University, Hamilton, ON, Canada in 2004, and is currently working toward the Ph.D. degree at the Department of Electrical Engineering, National University of Singapore. His research interests are in communication theory, broadband wireless communications, signal processing for communications, MIMO systems, and array signal processing.

Mr. Gao was a recipient of the president scholarship from the National University of Singapore. He has co-authored more than 30 refereed IEEE journal and conference papers and has served as a TPC member for IEEE ICC (2008), IEEE VTC (2008), and IEEE GLOBECOM (2008). 


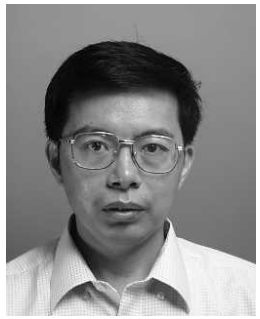

Yonghong Zeng (M'01-SM'05) Dr. Yonghong ZENG received the B.S. degree from the Peking University, Beijing, China, and the M.S. degree and the Ph.D. degree from the National University of Defense Technology, Changsha, China. He worked as an associate professor in the National University of Defense Technology before July 1999. From Aug. 1999 to Oct. 2004, he was a research fellow in the Nanyang Technological University, Singapore and the University of Hong Kong, successively. Since Nov. 2004, he has been working in the Institute for Infocomm Research, A*STAR, Singapore, as a scientist. His current research interests include signal processing and wireless communication, especially on cognitive radio and software defined radio, channel estimation, equalization, detection, and synchronization.

He has co-authored six books, including "Transforms and Fast Algorithms for Signal Analysis and Representation”, Springer-Birkhäuser, Boston, USA, 2003, and more than 60 refereed journal papers. He has four US patents. $\mathrm{He}$ is an IEEE senior member. He received the ministry-level Scientific and Technological Development Awards in China four times. In 2007, he received the IES prestigious engineering achievement award in Singapore.

He served as a TPC member for many prestigious international conferences such as IEEE ICC (2008), IEEE WCNC (2007, 2008), IEEE VTC (2008) and as the publication chair for CrownCom 2008.

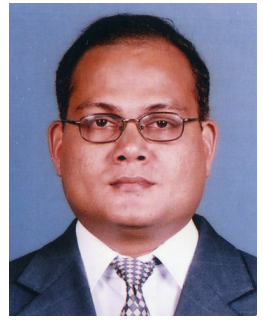

Arumugam Nallanathan (S'97-M'00-SM'05) received the B.Sc. with honors from the University of Peradeniya, Sri-Lanka, in 1991, the CPGS from the Cambridge University, United Kingdom, in 1994 and the Ph.D. from the University of Hong Kong, Hong Kong, in 2000, all in Electrical Engineering. He was an Assistant Professor in the Department of Electrical and Computer Engineering, National University of Singapore, Singapore from August 2000 to December 2007. Currently, he is a senior lecturer at King's College London. His research interests include OFDM systems, ultra-wide bandwidth (UWB) communication and localization, MIMO systems, and cooperative diversity techniques. In these areas, he has published over 100 journal and conference papers. He is a co-recipient of the Best Paper Award presented at 2007 IEEE International Conference on Ultra-Wideband.

He currently serves on the Editorial Board of IEEE Transactions on Wireless Communications, IEEE Transactions on Vehicular Technology, JohnWiley's Wireless Communications and Mobile computing and EURASIP Journal of Wireless Communications and Networking as an Associate Editor He served as a Guest Editor for EURASIP Journal of Wireless Communications and Networking: Special issue on UWB Communication Systems- Technology and Applications. He also served as a technical program committee member for more than 25 IEEE international conferences. He currently serves as the General Track Chair for IEEE VTC'2008-Spring, Co-Chair for the IEEE GLOBECOM'2008 Signal Processing for Communications Symposium, and IEEE ICC'2009 Wireless Communications Symposium.

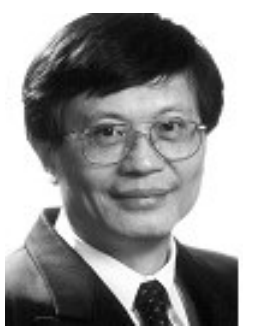

Tung-Sang Ng (S'74-M'78-SM'90-F'03) received the B.Sc.(Eng.) degree from The University of Hong Kong in 1972, the M.Eng.Sc. and Ph.D. degrees from the University of Newcastle, Australia, in 1974 and 1977, respectively, all in electrical engineering.

He worked for BHP Steel International and The University of Wollongong, Australia after graduation for 14 years before returned to The University of Hong Kong in 1991, taking up the position of Professor and Chair of Electronic Engineering. He was Head of Department of Electrical and Electronic Engineering from 2000 to 2003 and is currently Dean of Engineering. His current research interests include wireless communication systems, spread spectrum techniques, CDMA and digital signal processing.He has published over 250 international journal and conference papers.

He was the General Chair of ISCAS'97 and the VP-Region 10 of IEEE CAS Society in 1999 \& 2000. He was an Executive Committee Member and a Board Member of the IEE Informatics Divisional Board (1999-2001) and was an ordinary member of IEE Council (1999-2001).

He was awarded the Honorary Doctor of Engineering Degree by the University of Newcastle, Australia in 1997, the Senior Croucher Foundation Fellowship in 1999, the IEEE Third Millenium medal in 2000 and the Outstanding Researcher Award by The University of Hong Kong in 2003. $\mathrm{He}$ is a Fellow of IEE and HKIE. 